\title{
Gustatory dysfunctions in COVID-19
}

\author{
Jerome R. Lechien ${ }^{1,2,3,4}$. Julien W. Hsieh ${ }^{1,5} \cdot$ Tareck Ayad $^{1,6}$. Nicolas Fakhry ${ }^{1,7}$. Stephane Hans $\mathbf{s}^{1,3}$. \\ Carlos M. Chiesa-Estomba ${ }^{1,8} \cdot$ Sven Saussez ${ }^{2,4}$
}

Received: 10 June 2020 / Accepted: 17 June 2020 / Published online: 23 June 2020

c) Springer-Verlag GmbH Germany, part of Springer Nature 2020

Keywords Smell $\cdot$ Taste $\cdot$ Anosmia $\cdot$ Hyposmia $\cdot$ Gustatory $\cdot$ Dysgeusia $\cdot$ Loss $\cdot$ COVID-19 $\cdot$ Coronavirus $\cdot$ Dear editor

\section{Dear Editor,}

We thank Dr Bigiani [1] for this letter entitled "Gustatory dysfunctions in COVID-19 patients: possible involvement of taste renin-angiotensin system (RAS)" which is related to our paper [2]. Based on an experimental study reporting expression of Angiotensin Converting Enzyme-2 (ACE2) in taste organs of mouse, the author thinks that ACE2 could have a key role in the development of taste dysfunction in COVID-19 patients. Overall, the letter highlights the importance to consider gustatory impairment in COVID-19 patients.

Jerome R. Lechien and Julien W. Hsieh contributed to the paper and are joint as co-first authors.

Carlos M. Chiesa-Estomba and Sven Saussez contributed to the paper and are joint as co-senior authors.

This reply refers to the comment available online at https://doi. org/10.1007/s00405-020-06054-z.

Jerome R. Lechien

Jerome.Lechien@umons.ac.be

1 COVID-19 Task Force of the Young-Otolaryngologists of the International Federations of Oto-Rhino-

Laryngological Societies (YO-IFOS), Paris, France

2 Department of Human Anatomy and Experimental Oncology, Faculty of Medicine, UMONS Research Institute for Health Sciences and Technology, University of Mons (UMons), Mons, Belgium

3 Department of Otolaryngology-Head and Neck Surgery, Foch Hospital, School of Medicine, UFR Simone Veil, Université Versailles Saint-Quentin-en-Yvelines (Paris Saclay University), Paris, France

4 Department of Otorhinolaryngology and Head and Neck Surgery, CHU de Bruxelles, CHU Saint-Pierre, School of Medicine, Université Libre de Bruxelles, Brussels, Belgium
Since the outbreak of the pandemic, a particular attention has been paid to loss of smell, leaving out, in many studies, gustatory dysfunction. However, in clinical practice, COVID-19 patients report a significant impact of gustatory dysfunction in their quality of life. They typically report that things "tasted like cardboard". As proposed by Dr Bigiani, we believe that gustatory dysfunction, defined as the impairment of salty, sweet, bitter and sour, could be related to the virus spread into the nerve ending of the taste buds of the oral cavity. Pure taste disorders are rare and represent only $5 \%$ of specialized smell and taste consultation [3]. Upper respiratory tract infection is one of the most frequent suspected etiology in these cases, but remains largely under investigated [3]. In the particular case of COVID-19, ACE2 receptors has been identified in the oral cavity in humans with high expression level in the tongue [4], supporting the hypothesis that viral infection and inflammatory response may lead to disruption of saliva composition, normal taste transduction or the continuous renewal of taste buds [5].

5 Rhinology-Olfactology Unit, Department of Otorhinolaryngology, Head and Neck Surgery, Geneva University Hospitals (HUG), Geneva, Switzerland

6 Department of Otolaryngology-Head and Neck Surgery, CHU de Montreal, Montreal, Canada

7 Department of Otolaryngology-Head and Neck Surgery, APHM, Aix-Marseille Univ, Hopital La Conception, Marseille, France

8 Department of Otorhinolaryngology-Head and Neck Surgery, Hospital Universitario Donostia, San Sebastian, Spain 
Moreover, the virus is known to present a neurotropism, which may contribute to olfactory and gustatory dysfunction [6] and some neurological manifestations [7]. It could be possible that central lesions, caused by propagation of the virus through the olfactory pathways, lead to taste dysfunction. There is many overlapping brain areas between taste and olfactory system. One of them is the frontobasal regions, which is close to the olfactory bulbs that also express ACE2 receptors in their vascular pericytes. Inflammatory damage to this region may alter both senses, namely taste and olfactory function. The neurological damage caused by the virus is also supported by the findings of our last study, in which $20 \%$ of COVID-19 patients with anosmia did not recover within 2-month following the onset of olfactory dysfunction [8]. The lack of recovery of both taste and smell after weeks or months may support the occurrence of neurological damage due to extensive inflammation in these regions. Currently, there are few studies investigating the assessment of taste with electrophysiological or psychophysical tools. The prevalence of self-reported taste dysfunction, defined as altered perception of salty, sweet, bitter or/and sour, could reach $56.4 \%$ of patients [9]. However, we are aware that selfreported taste dysfunction might be unreliable and could be often confounded with loss of aroma perception, which is normally detected by the olfactory system. In contrast, patients who report having an intact sense of taste, usually do not have a deficit in taste function [10]. Despite the clear need for formal testing rather than self-reporting, we could hypothesize that at least half of the patients with COVID-19 should have an intact gustatory function.

The letter of Dr Bigiani and the current patient-reported outcome questionnaire findings of the literature [10,11] support the need to conduct future studies using at least psychophysical or electrophysiological taste evaluation by using Taste strips or electrogustometry [12] in COVID-19. The study of recovery rate of gustatory function is still important and has to be considered in these studies according to the substantial number of patients who report this issue in clinical practice. Future studies have to be carefully designed to discriminate smell, taste and altered aroma perception, which may be confounded by a large number of patients.

Funding This study (response to the editor) has not received any support from funding agencies.

\section{Compliance with ethical standards}

Conflict of interest The author had no conflict of interest.

Ethical approval This article does not contain any studies with human participants or animals performed by any of the authors.

\section{References}

1. Bigiani A (2020) Gustatory dysfunctions in COVID-19 patients: possible involvement of taste renin-angiotensin system (RAS). Eur Arch Otorhinolaryngol 20:1. https://doi.org/10.1007/s0040 5-020-06054-Z

2. Lechien JR, Chiesa-Estomba CM, De Siati DR et al (2020) Olfactory and gustatory dysfunctions as a clinical presentation of mildto-moderate forms of the coronavirus disease (COVID-19): a multicenter European study. Eur Arch Otorhinolaryngol. https://doi. org/10.1007/s00405-020-05965-1

3. Deems DA, Doty RL, Settle RG, Moore-Gillon V, Shaman P, Mester AF, Kimmelman CP, Brightman VJ, Snow JBJ (1991) Smell and taste disorders: a study of 750 patients from the University of Pennsylvania Smell and Taste Center. Arch Otorhinolaryngol Head Neck Surg. 117:519-528. https://doi.org/10.1001/archo tol.1991.01870170065015

4. Xu H, Zhong L, Deng J, Peng J, Dan H, Zeng X, Li T, Chen Q (2020) High expression of ACE2 receptor of 2019-nCoV on the epithelial cells of oral mucosa. Int J Oral Sci 12(1):8. https://doi. org/10.1038/s41368-020-0074-x

5. Wang H, Zhou M, Brand J, Huang L (2009) Inflammation and taste disorders: mechanisms in taste buds. Ann N Y Acad Sci 1170:596-603. https://doi.org/10.1111/j.1749-6632.2009.04480 .x

6. Lechien JR, Cabaraux P, Chiesa-Estomba CM et al (2020) Objective olfactory evaluation of self-reported loss of smell in a case series of 86 COVID-19 patients. Head Neck. https://doi. org/10.1002/hed.26279

7. Montalvan V, Lee J, Bueso T, De Toledo J, Rivas K (2020) Neurological manifestations of COVID-19 and other coronavirus infections: a systematic review. Clin Neurol Neurosurg 194:105921. https://doi.org/10.1016/j.clineuro.2020.105921

8. Lechien JR, Journe F, Beckers E et al. (2020) Severity of anosmia as early symptom of COVID-19 infection may predict lasting loss of smell. Clin Microbiol Infect (under review)

9. Lechien JR, Chiesa-Estomba CM, Hans S, Barillari MR, Jouffe L, Saussez S (2020) Loss of smell and taste in 2013 European patients with mild to moderate COVID-19. Ann Intern Med. https ://doi.org/10.7326/M20-2428

10. Soter A, Kim J, Jackman A, Tourbier I, Kaul A, Doty RL (2008) Accuracy of self-report in detecting taste dysfunction. Laryngoscope 118(4):611-617. https://doi.org/10.1097/MLG.0b013e3181 $61 \mathrm{e} 53 \mathrm{a}$

11. Vaira LA, Hopkins C, Salzano G et al (2020) Olfactory and gustatory function impairment in COVID-19 patients: Italian objective multicenter-study. Head Neck. https://doi.org/10.1002/hed.26269

12. Pavlidis P, Gouveris C, Kekes G, Maurer J (2014) Changes in electrogustometry thresholds, tongue tip vascularization, density and form of the fungiform papillae in smokers. Eur Arch Otorhinolaryngol 271(8):2325-2331. https://doi.org/10.1007/s0040 5-014-3003-9

Publisher's Note Springer Nature remains neutral with regard to jurisdictional claims in published maps and institutional affiliations. 